\title{
Implied distribution as a function of the volatility smile
}

\author{
Bertrand TAVIN * \\ Université Paris 1 - Panthéon Sorbonne (Laboratoire PRISM) \\ bertrand.tavin@univ-paris1.fr
}

June 1, 2011

\begin{abstract}
The aim of this paper is to obtain the risk-neutral density of an underlying asset price as a function of its option implied volatility smile. We derive a known closed form non-parametric expression for the density and decompose it into a sum of lognormal and adjustment terms. By analyzing this decomposition we also derive two no-arbitrage conditions on the volatility smile. We then explain how to use the results. Our methodology is applied first to the pricing of a portfolio of digital options in a fully smile-consistent way. It is then applied to the fitting of a parametric distribution for log-return modelling, the Normal Inverse Gaussian.
\end{abstract}

Keywords : Option pricing, Risk-neutral distribution, Implied volatility smile.

JEL Classification : C14, C52, G13.

EFM Classification : 410,450 .

\section{Introduction}

The knowledge of the risk-neutral density of an asset price is of great interest for researchers and market practitioners. It has applications such as option pricing and analysis of market information. Following the seminal result of Breeden and Litzenberger in [7] (BREEDEN \& LitZENBERGER, 1978) the study of risk-neutral densities is the topic of an important literature.

The great majority of this literature details approaches where risk-neutral density is obtained from option prices. See among others [19] (Jackwerth \& Rubinstein, 1996) [2] (AÏT-SAhalia \& Lo, 1998) and [13] (Figlewski, 2010). See also [18] (JACKWERTh, 2004) for a detailed review of literature and methodologies. In [6] (Bliss \& PANigirtzoglou, 2002) authors recommend an intermediary step where prices are converted to implied volatility to be smoothed and then converted back to prices for the density to be computed. [23] (Kermiche, 2009) uses this approach to construct the evolution over time of the risk-neutral density implied in CAC40 options and performs a principal component analysis to study its behavior.

Our approach to compute risk-neutral density is much simpler because it involves only one step as we consider implied volatility as the available market data for options. Monitoring an options market through implied volatility can be seen as more convenient than doing it through prices. A first reason is that implied volatility can be instantly compared across strikes, where prices need to be adjusted for the underlying price before being compared. Another reason is that, on some markets, options are negotiated in terms of implied volatility instead of price. For example this convention is in use for over-the-counter currency options. See [24] (LEE, 2005) who also advocates using implied volatility to represent options data.

\footnotetext{
${ }^{*}$ Acknowledgments : I am grateful to my PhD supervisors Prof. Patrice PONCET (ESSEC Business School) and Prof. Constantin MELLIOS (Université Paris 1) for their comments and helpful discussions. I also thank Peter CARR, Laurent DEVILLE, Jens JACKWERTH, Franck MORAUX and an anonymous referee for their comments on earlier versions. All remaining errors are mine. First version : November 3, 2010. Contact: Laboratoire PRISM - EA4101 17 rue de la Sorbonne 75005 Paris France, Tel: +33140463170 Fax: +33140463011
} 
In this paper we propose a methodology to directly build a risk-neutral density from implied volatility data. We take the Breeden-Litzenberger formula, see [7] (BREEDEN \& LitzEnBERGER, 1978), as starting point and derive a known result that can be seen as an implied volatility version of it. This result has already been obtained in [17] (JACKWERTH, 2000) and [8] (BRUnNER \& HAFNER, 2003).

It is possible to obtain this implied volatility version because there is a one to one relation between option price and implied volatility. This one to one relation is Black-Scholes formula, see [5] (Black \& Scholes, 1973), or Black's formula, see [4] (Black, 1976), both seen as functions of the volatility parameter $\sigma$ (other parameters and variables held constant).

The paper is organized as follows. In section 2 we first explain the financial setup. We then propose an intuitive and closed form decomposition of the underlying asset price's risk-neutral density as a function of the volatility smile and make comments on its terms. Finally, we examine how to use the formula and provide a numerical example. In section 3 we apply our method to the pricing of a portfolio of digital options. We then study how to fit a parametric distribution for log returns, the Normal Inverse Gaussian (NIG), directly to the volatility smile using the formula and avoiding the computation of option prices. In section 4 we conclude.

\section{Implied density of an underlying asset price}

\subsection{Financial framework}

We consider a financial market in continuous time where an asset $S$ is traded. We consider that $S$ pays and instantaneous dividend rate. We denote $S_{t}$ and $\delta_{t}$ its time $t$ price and dividend rate. European vanilla options on $S$ are also traded. A strike $K$, maturity $T$, vanilla option pays at time $T$

$$
\begin{aligned}
& C_{T}=\left(S_{T}-K\right)^{+} \text {for a Call option } \\
& P_{T}=\left(K-S_{T}\right)^{+} \text {for a Put option }
\end{aligned}
$$

We consider that a money market and zero-coupon bonds are available for trading. We denote $n(t)$ the time $t$ value of the money market account (with $n(0)=1$ ) and $B(t, T)$ the time $t$ price of the $\mathrm{ZC}$ bond maturing at time $T$. The money market account is an asset earning the instantaneous risk-free rate, which is assumed to follow a stochastic process $r_{t}(t \geq 0)$ :

$$
n(t)=\exp \left(\int_{0}^{t} r_{s} d s\right)
$$

As we suppose that our market is free of arbitrage, there exists a risk-neutral probability $\mathbb{Q}$ equivalent to the true (historical) probability $\mathbb{P}$. This probability is associated with $(n(t), t \geq 0)$ as numéraire. Asset prices can be written as expectations, under $\mathbb{Q}$, of their discounted payoffs.

$$
\begin{gathered}
B(0, T)=\mathbb{E}^{\mathbb{Q}}\left[\frac{1}{n(T)}\right]=\mathbb{E}^{\mathbb{Q}}\left[\exp \left(-\int_{0}^{T} r_{s} d s\right)\right] \\
C_{0}^{*}=\mathbb{E}^{\mathbb{Q}}\left[\frac{C_{T}}{n(T)}\right]=\mathbb{E}^{\mathbb{Q}}\left[\exp \left(-\int_{0}^{T} r_{s} d s\right)\left(S_{T}-K\right)^{+}\right] \\
P_{0}^{*}=\mathbb{E}^{\mathbb{Q}}\left[\frac{P_{T}}{n(T)}\right]=\mathbb{E}^{\mathbb{Q}}\left[\exp \left(-\int_{0}^{T} r_{s} d s\right)\left(K-S_{T}\right)^{+}\right]
\end{gathered}
$$

When interest rates are stochastic it is more convenient to work with the forward-neutral probability $\mathbb{Q}_{T}$ associated with $T$, the maturity of the European options. $\mathbb{Q}_{T}$ is the probability 
associated with $(B(t, T), t \geq 0)$ as numraire:

$$
\begin{gathered}
\frac{d \mathbb{Q}_{T}}{d \mathbb{Q}}=\frac{B(T, T)}{B(0, T)} \frac{n(0)}{n(T)}=\frac{1}{B(0, T) n(T)} \\
C_{0}^{*}=B(0, T) \mathbb{E}^{\mathbb{Q}_{T}}\left[C_{T}\right]=B(0, T) \mathbb{E}^{\mathbb{Q}_{T}}\left[\left(S_{T}-K\right)^{+}\right] \\
P_{0}^{*}=B(0, T) \mathbb{E}^{\mathbb{Q}_{T}}\left[P_{T}\right]=B(0, T) \mathbb{E}^{\mathbb{Q}_{T}}\left[\left(K-S_{T}\right)^{+}\right]
\end{gathered}
$$

For details on this change of probability, see chap. 11 and 19 in [27] (PorTAit \& PonCET, 2010). In the sequel, we work with undiscounted option prices, defined as

$$
C_{0}=\frac{C_{0}^{*}}{B(0, T)}=\mathbb{E}^{\mathbb{Q}_{T}}\left[\left(S_{T}-K\right)^{+}\right] \quad P_{0}=\frac{P_{0}^{*}}{B(0, T)}=\mathbb{E}^{\mathbb{Q}_{T}}\left[\left(K-S_{T}\right)^{+}\right]
$$

\subsection{Expression and decomposition of the implied density}

Considering a vanilla option price as a function of strike and maturity, the Breeden-Litzenberger formula links $\phi_{T}$, the risk-neutral density of $S_{T}$, with a second order partial derivative of this function. For a fixed $T>0$, and $\forall k \geq 0$

$$
\phi_{T}(k)=\frac{1}{B(0, T)} \frac{\partial^{2} C^{*}}{\partial K^{2}}(k, T)=\frac{1}{B(0, T)} \frac{\partial^{2} P^{*}}{\partial K^{2}}(k, T)
$$

This formula is key to obtain Dupire's equation for local volatility models, see [11] (DupIRE, 1993) or to reveal anticipations of market participants embedded in option prices, see [20] (Jondeau \& Rockinger, 2000). With undiscounted option prices, $C$ and $P$, the formula simplifies to

$$
\phi_{T}(k)=\frac{\partial^{2} C}{\partial K^{2}}(k, T)=\frac{\partial^{2} P}{\partial K^{2}}(k, T)
$$

In our stochastic interest rates setting $\phi_{T}$ is the density of $S_{T}$ under $\mathbb{Q}_{T}$ the $T$-forward neutral probability. When rates are deterministic, the forward and risk-neutral probabilities are identical, that is $\mathbb{Q}_{T}=\mathbb{Q} \quad \forall T \geq 0$.

Whether interest rates are deterministic or not does not matter here. Breeden-Litzenberger formula gives the underlying density under the relevant pricing measure. So that the price of a European claim $v$ paying $h\left(S_{T}\right)$ at time $T$ can always be written as

$$
v_{0}^{*}=B(0, T) \int_{0}^{+\infty} h(x) \phi_{T}(x) d x=B(0, T) \int_{0}^{+\infty} h(x) \frac{\partial^{2} C}{\partial K^{2}}(x, T) d x
$$

A maturity $T$, strike $K$, vanilla option on $S$ can be seen as an option on $F^{T}$, the forward contract written on $S$ and expiring at time $T$. As $S_{T}=F_{T}^{T}$, we have

$$
C_{T}=\left(S_{T}-K\right)^{+}=\left(F_{T}^{T}-K\right)^{+} \quad P_{T}=\left(K-S_{T}\right)^{+}=\left(K-F_{T}^{T}\right)^{+}
$$

The time $t$ forward price of $S$ is (with $t \in[0, T]$ )

$$
F_{t}^{T}=\frac{S_{t} \exp \left(-\int_{t}^{T} \delta_{u} d u\right)}{B(t, T)}
$$


It is a martingale under $\mathbb{Q}_{T}$.

Under the assumption of a constant volatility geometric Brownian motion diffusion for $F$, Black's formula holds, see [4] (BLACK, 1976). The undiscounted call price given by Black's formula at time $t=0$ is

$$
\begin{gathered}
C_{B}(F, \sigma, K, T)=F \mathcal{N}\left(d_{1}\right)-K \mathcal{N}\left(d_{0}\right) \\
d_{0}(F, \sigma, K, T)=\frac{\ln \frac{F}{K}}{\sigma \sqrt{T}}-\frac{1}{2} \sigma \sqrt{T} \quad d_{1}=d_{0}+\sigma \sqrt{T}
\end{gathered}
$$

where $\mathcal{N}$ is the cumulative distribution function of a standard Gaussian:

$$
\mathcal{N}: x \longmapsto \mathcal{N}(x)=\frac{1}{\sqrt{2 \pi}} \int_{-\infty}^{x} \exp \left(-\frac{t^{2}}{2}\right) d t
$$

This function is not known in closed form but fast and accurate approximations are available, see for example Chap. 26 in [1] (Abramovitz \& Stegun, 1972).

Black's formula seen as a function of the volatility $\left(\sigma \longmapsto C_{B}(F, \sigma, K, T)\right)$ is strictly increasing. Hence from any observed call price it is possible to numerically back out a unique implied volatility parameter by inverting this function. Observed option prices are market prices or model outputs.

Practitioners represent vanilla options market data as implied volatility because it is easier to interpret and to monitor. For a given maturity, implied volatility as a function of strike is not constant and often smile shaped or skewed. It is usually called volatility smile. Although this fact invalidates the assumption behind Black's formula, it is still possible to use it to express observed prices with a strike dependent volatility parameter

$$
C_{o b s}(K, T)=C_{B}(F, \sigma(K, T), K, T)
$$

In the right hand side of (2.14) the strike dependence is twofold. Plugging this representation of observed prices into formula (2.10) should allow us to obtain the risk-neutral density of $S_{T}$ as a function of the volatility smile. The second order derivative of $C$ with respect to $K$ will be expressed with partial derivatives of Black's formula with respect to $K$ and $\sigma$. Applying twice the chain rule for partial derivatives leads to

$$
\begin{gathered}
\frac{\partial C}{\partial K}=\frac{\partial C_{B}}{\partial K}+\frac{\partial C_{B}}{\partial \sigma} \frac{\partial \sigma}{\partial K} \\
\phi_{T}=\frac{\partial^{2} C}{\partial K^{2}}=\frac{\partial^{2} C_{B}}{\partial K^{2}}+2 \frac{\partial^{2} C_{B}}{\partial \sigma \partial K} \frac{\partial \sigma}{\partial K}+\frac{\partial^{2} C_{B}}{\partial \sigma^{2}}\left(\frac{\partial \sigma}{\partial K}\right)^{2}+\frac{\partial C_{B}}{\partial \sigma} \frac{\partial^{2} \sigma}{\partial K^{2}}
\end{gathered}
$$

Partial derivatives of $C_{B}$ involved in (2.16) are known in closed form. They are similar to the Greeks in Black's model: Gamma and Vanna with respect to strike, Vomma (also called Volga) and Vega.

$$
\begin{array}{ccrl}
\frac{\partial^{2} C_{B}}{\partial K^{2}}=\frac{n\left(d_{0}\right)}{K \sigma \sqrt{T}} & \frac{\partial^{2} C_{B}}{\partial \sigma \partial K}=\frac{n\left(d_{0}\right) d_{1}}{\sigma} \\
\frac{\partial^{2} C_{B}}{\partial \sigma^{2}}=\frac{d_{0} d_{1}}{\sigma} n\left(d_{1}\right) F \sqrt{T} & \frac{\partial C_{B}}{\partial \sigma}=n\left(d_{1}\right) F \sqrt{T}
\end{array}
$$

where $n=\frac{\partial \mathcal{N}}{\partial x}$ is the probability density function of a standard Gaussian.

$$
n: x \longmapsto n(x)=\frac{1}{\sqrt{2 \pi}} \exp \left(-\frac{x^{2}}{2}\right)
$$


See Appendix A.1 for derivation details.

As $K \longmapsto \sigma(K, T)$ is the smile function, partial derivatives of $\sigma$ involved in (2.16) make it dependent of the volatility smile, unsurprisingly. Graphically they correspond to the smile slope, convexity and squared slope. It is interesting to remark that only the first two derivatives of the smile are needed to express the risk-neutral density. We define now $G=G(K, T)$ and $H=H(K, T)$ as the smile slope and convexity (with respect to strike) at point $(K, T)$.

$$
\frac{\partial \sigma}{\partial K}=G \quad \frac{\partial^{2} \sigma}{\partial K^{2}}=H
$$

Substituting (2.17), (2.18) and (2.19) in (2.16) we get the density as a closed form nonparametric function of the implied volatility smile

$$
\phi_{T}=\frac{n\left(d_{0}\right)}{K \sigma \sqrt{T}}+2 G \frac{n\left(d_{0}\right) d_{1}}{\sigma}+\left(G^{2} \frac{d_{0} d_{1}}{\sigma}+H\right) n\left(d_{1}\right) F \sqrt{T}
$$

This formula is also derived in [17] (JACKWERTH, 2000) where it is used to obtain risk aversion functions, and in [8] (BRUNNER \& HAFNER, 2003) where it is used in the context of a no-arbitrage analysis of the volatility smile.

We now rearrange the above formula to propose a decomposition of $\phi_{T}$ as a lognormal density term $f$ plus two adjustment terms $A_{1}$ and $A_{2}$.

$$
\phi_{T}(k)=f(k)+A_{1}(k)+A_{2}(k) \quad k>0
$$

Where $f$ is a probability density function, which corresponds to the lognormal density of $S_{T}$ in Black's model with a volatility parameter equal to $\sigma_{0}=\sigma(F, T)$, the ATM implied volatility (an option is said At The Money if struck at $F$ ). $A_{1}$ is a level adjustment term, accounting for the difference between ATM implied volatility $\sigma_{0}$ and its value at strike. And $A_{2}$ is also an adjustment term, accounting for the slope and convexity of the smile. These terms are written

$$
\begin{aligned}
f(k) & =f_{L N}\left(k ; \ln F-\frac{1}{2} \sigma_{0}^{2} T, \sigma_{0}^{2} T\right) \\
A_{1}(k) & =\frac{1}{k \sqrt{T}}\left(\frac{1}{\sigma(k)} n\left(d_{0}(F, \sigma(k), k, T)\right)-\frac{1}{\sigma_{0}} n\left(d_{0}\left(F, \sigma_{0}, k, T\right)\right)\right) \\
A_{2}(k) & =2 G(k) \frac{n\left(d_{0}\right) d_{1}}{\sigma(k)}+\left(G(k)^{2} \frac{d_{0} d_{1}}{\sigma(k)}+H(k)\right) n\left(d_{1}\right) F \sqrt{T}
\end{aligned}
$$

The function $x \longmapsto f_{L N}\left(x ; m, s^{2}\right)$ is the density function of a lognormal distribution with parameters $m$ and $s^{2}$. That is, $\forall x>0$

$$
f_{L N}\left(x ; m, s^{2}\right)=\frac{1}{x s \sqrt{2 \pi}} \exp \left(-\frac{1}{2}\left(\frac{\ln x-m}{s}\right)^{2}\right)
$$

Option traders usually analyze the implied volatility smile in three steps, examining well known trading strategies. The first step is to check the ATM level, kept as a reference. In terms of options trading it usually corresponds to an ATM straddle ${ }^{1}$ strategy. The second is to compare

\footnotetext{
${ }^{1}$ A long ATM Straddle involves a call and a put, both long and struck at $F$.
} 
the implied volatility at a specified strike $K$ with the ATM reference. It corresponds to a call spread $^{2}$ (or put spread) strategy with one option struck at $F$ and the other at $K$. The third step is to analyze the overall shape of the volatility smile, its slope and convexity. That is done with a risk reversal strategy ${ }^{3}$ for the slope and with a butterfly ${ }^{4}$ for the convexity.

The decomposition (2.21) of the density as a closed form function of the smile is sound and makes sense financially because it is built exactly on the same three steps.

Pursuing further the analysis of (2.21) we derive no-arbitrage conditions on adjustment terms. For $\phi_{T}$ to be a proper probability density function it should integrate to 1 on $\mathbb{R}^{+}$and be positive everywhere. That is

$$
\begin{aligned}
\int_{0}^{+\infty} \phi_{T}(k) d k & =1 \\
\phi_{T}(k) & \geq 0 \forall k \geq 0
\end{aligned}
$$

This constraint on the density function is used in [8] (BRUnner \& HAFner, 2003). See also [24] (LEE, 2005) who provides no-arbitrage bounds on the slope of the volatility smile.

As $f$ already is a probability density function, it verifies

$$
\begin{aligned}
\int_{0}^{+\infty} f(k) d k & =1 \\
f(k) & \geq 0 \quad \forall k \geq 0
\end{aligned}
$$

Hence we bring out two no-arbitrage conditions on the volatility smile. The first one is global. The second one is local and holds at any particular point.

$$
\begin{gathered}
\int_{0}^{+\infty}\left(A_{1}(k)+A_{2}(k)\right) d k=0 \\
A_{1}(k)+A_{2}(k) \geq-f(k) \quad \forall k \geq 0
\end{gathered}
$$

The particularity of the above two results is to link at the same time the level, slope and convexity of the volatility smile.

\subsection{Working with the formula}

To work with the risk-neutral density formula (2.21) one needs implied volatility values at any positive strike. Market data for traded options is only available at discrete strike points. The implied volatility smile corresponding to real market data is a set of points instead of a continuous curve. So we need an interpolation/extrapolation engine for the implied volatility : that is a technique to produce a continuous smile, given a discrete market data set.

We consider three different techniques, which correspond to different approaches to smile generation. Many techniques are acceptable for that purpose. We choose to present and implement three classical techniques. The first smile generation technique we consider is a parametrization based on the implied volatility formula derived in stochastic volatility SABR model, introduced by [16] (HAGAN et al., 2002). We take the version of the formula proposed in [26] (OBLOJ, 2008) because it has less occurrence of arbitrage at very low strikes than Hagan's version. This parametrization works with 3 parameters $\left(\alpha_{0}, \rho, v\right)$ plus a fixed fourth parameter $\beta$.

\footnotetext{
${ }^{2}$ A Call Spread involves two calls, a long and a short, with different strikes.

${ }^{3}$ A Risk Reversal involves a long call, struck higher than $F$, and a short put, struck lower than $F$.

${ }^{4} \mathrm{~A}$ long Butterfly involves two long calls, struck higher and lower than $F$, and two short calls, struck at $F$.
} 
Under SABR parametrization, implied volatility is written, for $K \neq F$

$$
\sigma_{S A B R}(K, F)=\frac{v \ln \left(\frac{F}{K}\right)}{\ln \left(\frac{\sqrt{1-2 \rho z+z^{2}}+z-\rho}{1-\rho}\right)}\left(1+T\left(\frac{1}{24} \frac{(1-\beta)^{2} \alpha_{0}^{2}}{(F K)^{(1-\beta)}}+\frac{1}{4} \frac{\rho \beta \alpha_{0} v}{(F K)^{\frac{1}{2}(1-\beta)}}+\frac{2-3 \rho^{2}}{24} v^{2}\right)\right)
$$

and for $K=F$

$$
\sigma_{S A B R}(F, F)=\frac{\alpha_{0}}{F^{1-\beta}}\left(1+T\left(\frac{1}{24} \frac{(1-\beta)^{2} \alpha_{0}^{2}}{F^{2(1-\beta)}}+\frac{1}{4} \frac{\rho \beta \alpha_{0} v}{F^{(1-\beta)}}+\frac{2-3 \rho^{2}}{24} v^{2}\right)\right)
$$

where

$$
z=\frac{v}{\alpha_{0}} \frac{F^{(1-\beta)}-K^{(1-\beta)}}{1-\beta}
$$

The second technique we consider is the SVI parametrization. It is an on purpose(ad hoc) functional form for the smile proposed in [15] (GATHeRAL, 2004). SVI stands for Stochastic Volatility Inspired. This parametrization is arbitrage free and works with 5 parameters $(a, b, v, \rho, m)$. Under SVI parametrization, implied volatility is written

$$
\sigma_{S V I}(K, F)=\frac{1}{\sqrt{T}} \sqrt{a+b\left(\rho\left(\ln \frac{K}{F}-m\right)+\sqrt{\left(\ln \frac{K}{F}-m^{2}+v^{2}\right)}\right)}
$$

SABR and SVI parametrizations need to be calibrated to the available market smile points. It is done by minimizing the sum of squared errors on volatility values using a deterministic, gradient based, optimization routine (Levenberg-Marquardt algorithm). We consider that market data is available for a number $N$ of strikes and we denote $\sigma_{M}\left(K_{i}\right)$ the corresponding set of volatility values $(i=1, \ldots, N)$.

Calibration is done by solving the following minimization programs

$$
\begin{gathered}
\mathcal{P}_{1}: \min _{(\alpha, \rho, v)} \sum_{i=1}^{N}\left(\sigma_{S A B R}\left(K_{i}\right)-\sigma_{M}\left(K_{i}\right)\right)^{2} \\
\mathcal{P}_{2}: \min _{(a, b, v, \rho, m)} \sum_{i=1}^{N}\left(\sigma_{S V I}\left(K_{i}\right)-\sigma_{M}\left(K_{i}\right)\right)^{2}
\end{gathered}
$$

The last technique we consider is a non-parametric regression, where the smile is built using the Nadaraya-Watson Kernel estimator (NWK). This technique is commonly used in nonparametric statistics. It has been presented and detailed in the context of implied distributions by [2] (Aїт-Sahalia \& Lo, 1998) and it used for example in [23] (Kermiche, 2009). Implied volatility at strike $K$ is written as a weighted average of the available data points

$$
\sigma_{N W K}(K)=\frac{\sum_{i=1}^{N} Z\left(\frac{K-K_{i}}{h}\right) \sigma_{M}\left(K_{i}\right)}{\sum_{i=1}^{N} Z\left(\frac{K-K_{i}}{h}\right)}
$$

where $Z$ is a kernel function (positive, symmetric and integrating to 1 ) and $h$ is the bandwidth parameter controlling for smoothness of the obtained function.

We choose to work with the classical Gaussian kernel, so that $Z$ actually is the Gaussian probability density function. The value of the smoothing parameter $h$ affects the smoothness 
of the obtained curve (higher value for more smoothness). It affects as well the data fit quality (lower value for better fit), so that the choice of a value for $h$ boils down to a trade-off between both. See [2] (Aїт-Sahalia \& Lo, 1998) for details on how to compute $h$.

The kernel regression method needs no calibration, as the volatility at any strike is obtained directly as a weighted average of market data.

We now consider a numerical illustration of the proposed method. The underlying is the DJ Eurostoxx 50, it is an equity index composed of 50 large eurozone companies. The details of market data are summarized in table 1 below. In the sequel, forward value is normalized at 100.

\begin{tabular}{|c||c|}
\hline Underlying & SX5E Index \\
\hline Date & $20-12-2007$ \\
\hline$S_{t}$ & 4315 \\
\hline$T$ & 1 year \\
\hline$F_{t}^{T}$ & 4350 \\
\hline$B(t, T)$ & 0.9547 \\
\hline$N$ & 13 \\
\hline
\end{tabular}

Table 1: Market data details

Figure 1 shows implied volatility market data points and the smile curves produced with fitted smile generation engines. It can be noted that between data points smile curves are close to each other, but away from data points differences are noticeable and due to the different behaviors of parametrization methods.

Figure 2 shows the risk-neutral density obtained using the proposed method (with the different smile engines). Lognormal density corresponding to Black's model is plotted as a reference. Obtained densities are smooth and close to each other, with differences near their maxima. They are clearly skewed to the left compared to the lognormal one.

Figure 3 shows the total adjustment term $A_{1}+A_{2}$ and the no-arbitrage bound corresponding to the local condition (2.23). The three plotted curves are above the no-arbitrage bound, illustrating that the condition is met.

Figures 4 and 5 show the details of adjustment terms $A_{1}$ and $A_{2}$ respectively. Curves on both figures are smooth and it can be noted that the order of magnitude of the two adjustment terms is the same. 


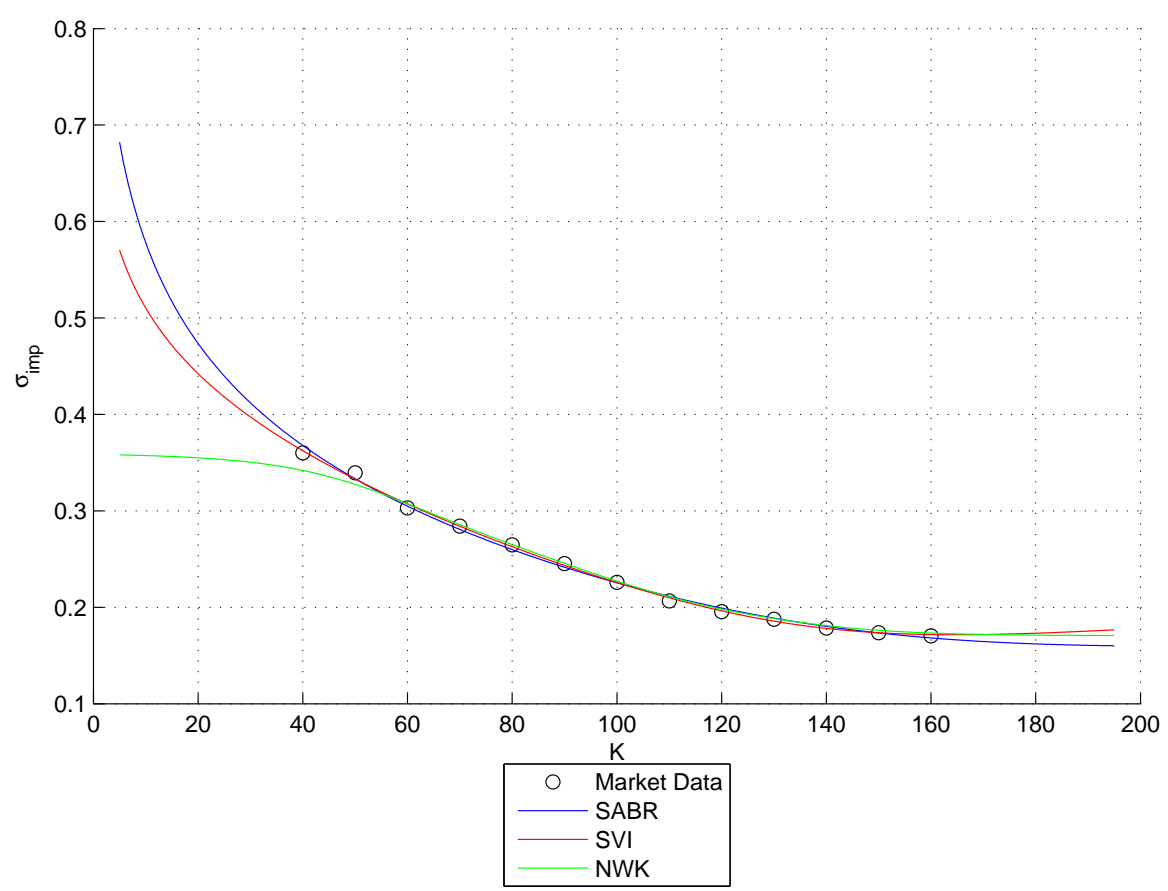

Figure 1: SX5E Implied Volatility Smile $(T=1$ year, $F=100, N=13)$

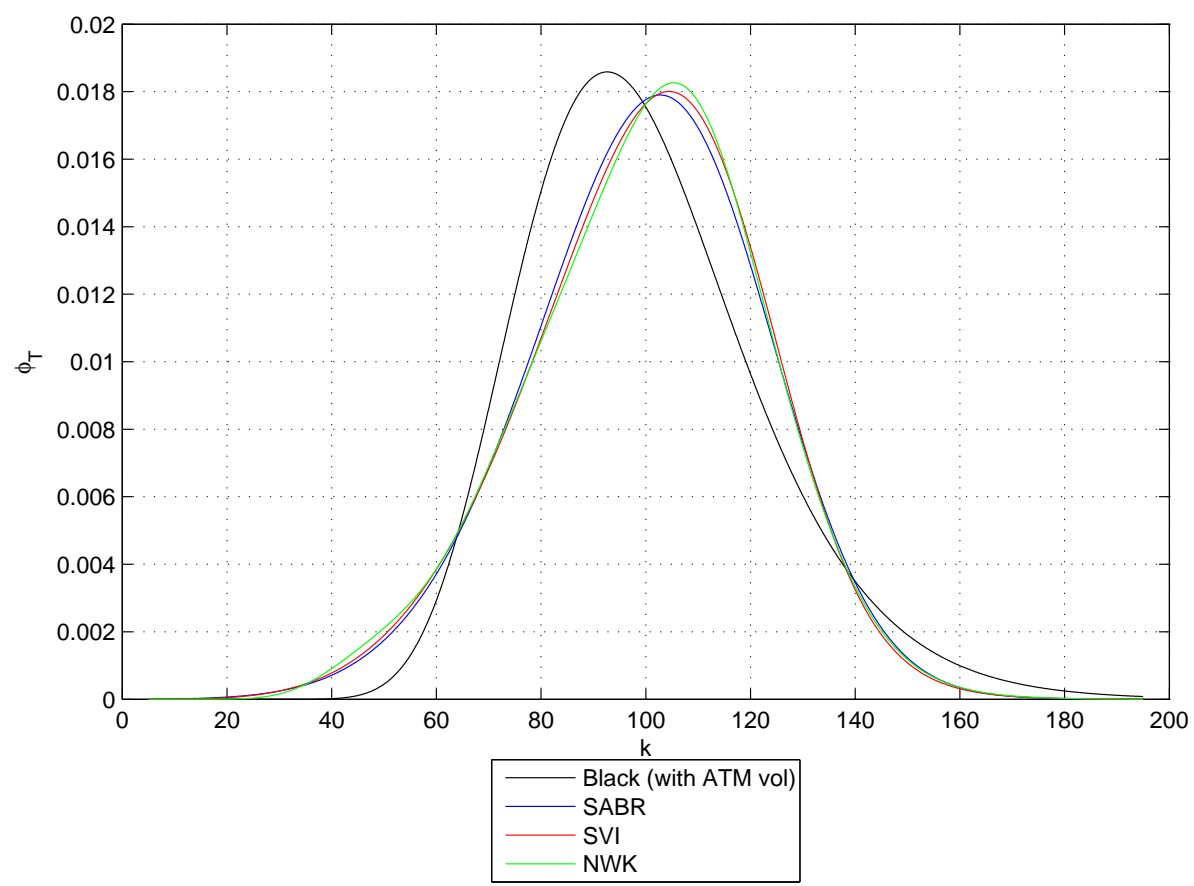

Figure 2: Risk-Neutral Density 


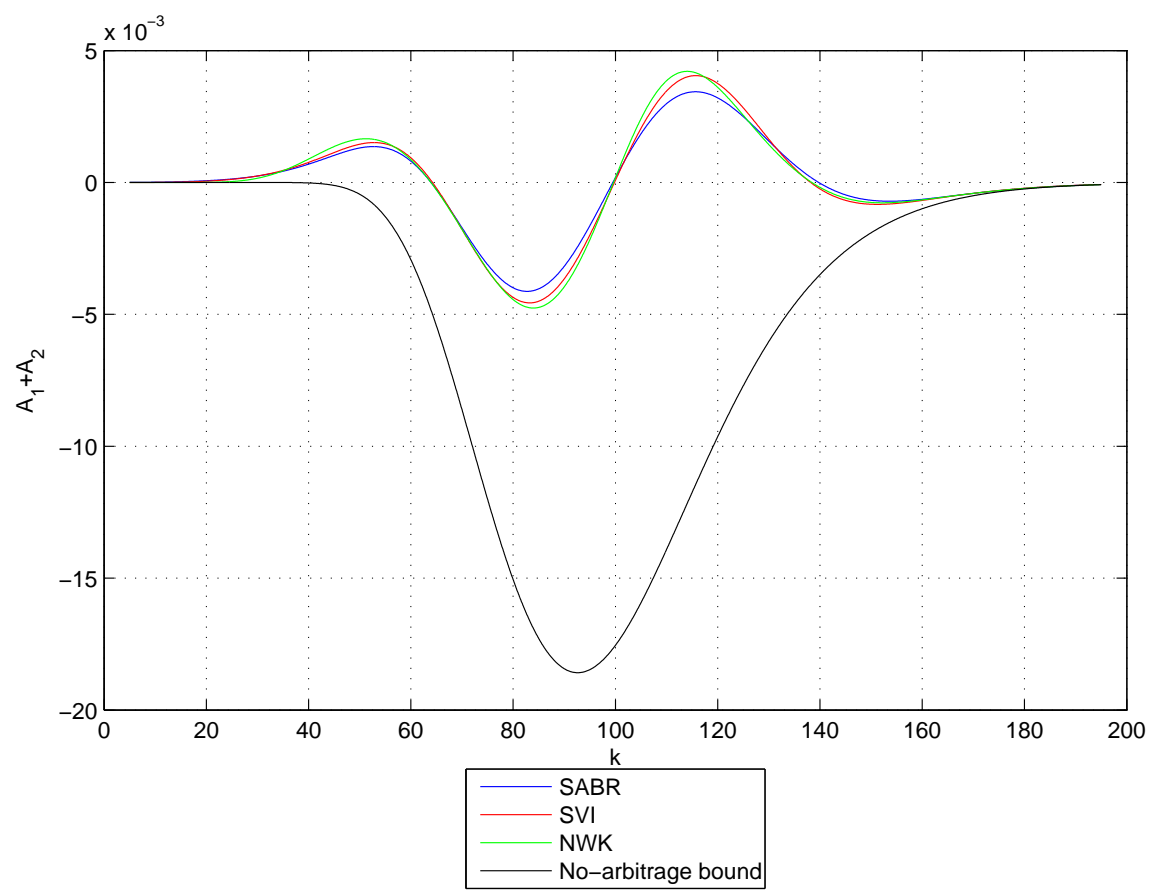

Figure 3: Total Adjustement Term

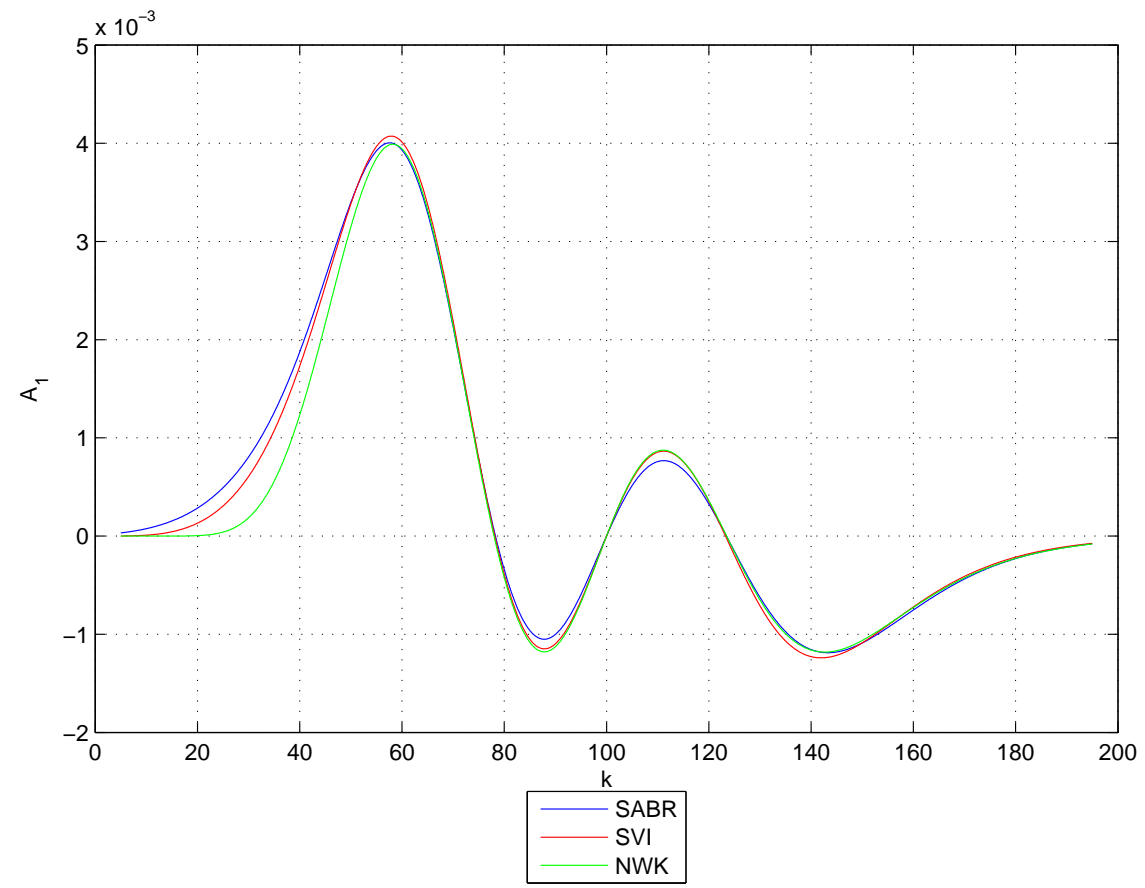

Figure 4: Level Adjustment Term 


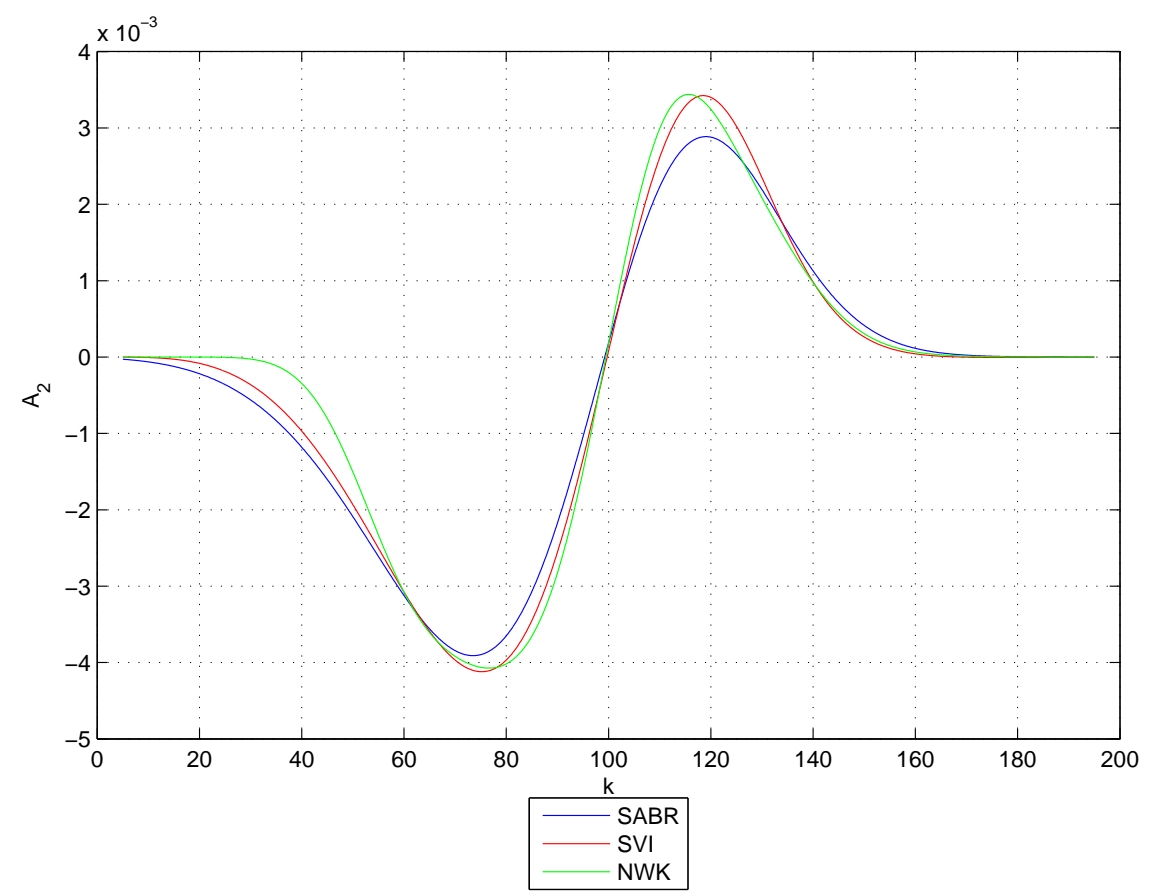

Figure 5: Shape Adjustment Term

\section{Applications}

Our approach to expressing the risk-neutral density, detailed in sections 2 and 2.3, is now applied to concrete cases. The first case we present is the pricing of a portfolio of digital options on $S$ in a fast and fully smile-consistent way. In the second we model the log-return of $S$ using a parametric distribution, the Normal Inverse Gaussian, and fit it to the non-parametric riskneutral distribution obtained from the volatility smile.

\subsection{Pricing of digital options}

A European, maturity $T$, digital call (put) option is an option paying 1 at time $T$ if $S_{T}$ is above (below) $K$, its strike, and 0 otherwise.

$$
\begin{aligned}
& D C_{T}=\left\{S_{T} \geq K\right\} \text { for a digital call } \\
& D P_{T}=\left\{S_{T} \leq K\right\} \text { for a digital put }
\end{aligned}
$$

Digital options are known to be sensitive not only to the implied volatility level at strike but also to the shape of the volatility smile, see Chap. 17 in [28] (TALEB, 1997). Using a smiled Black model to price it (a formula with a strike dependent volatility) is not appropriate because the whole shape of the smile is not accounted for. The undiscounted price of a digital call option $D C_{0}$ is obtained as

$$
\begin{gathered}
D C_{0}^{*}=B(0, T) \mathbb{E}^{\mathbb{Q}_{T}}\left[\left\{S_{T} \geq K\right\}\right]=B(0, T) \mathbb{Q}_{T}\left(\left\{S_{T} \geq K\right\}\right) \\
D C_{0}=\mathbb{Q}_{T}\left(\left\{S_{T} \geq K\right\}\right)=\int_{K}^{+\infty} \phi_{T}(k) d k
\end{gathered}
$$

It can also be written directly using (2.11) with $h\left(S_{T}\right)=\left\{S_{T} \geq K\right\}$

$$
D C_{0}=\int_{0}^{+\infty}\{k \geq K\} \phi_{T}(k) d k=\int_{K}^{+\infty} \phi_{T}(k) d k
$$


Our method is particularly suitable for handling large portfolios of digital options because it allows for a systematic pricing, consistent with the full smile. To obtain digital option prices it is necessary to compute the integral in (3.2). A numerical quadrature method is needed because $\phi_{T}$, while known, has no parametric form. In our numerical implementation we use a GaussKronrod adaptative quadrature method. This method is appropriate because it is robust and allows for a control of error.

Figure 6 below shows the undiscounted prices of digital calls on $S$. Results obtained with the 3 different smile interpolation techniques are plotted. Prices corresponding to Black's model (with volatility input equals to ATM value) are also shown as a reference. Market data is identical as in Part 2.3.

As expected, prices are substantially different from prices obtained with Black's model, even for the ATM struck option, because of the smile shape. Error made using Black's price is not negligible, it is positive or negative depending on the option's strike.

Curves obtained are close to each other illustrating the stability across strikes of our method to price digital options.

To plot figure 6, digital calls were priced (2000 different strikes) with 3 smile interpolation methods: the computation is done in less than 20 seconds with a Matlab program on a personal computer.

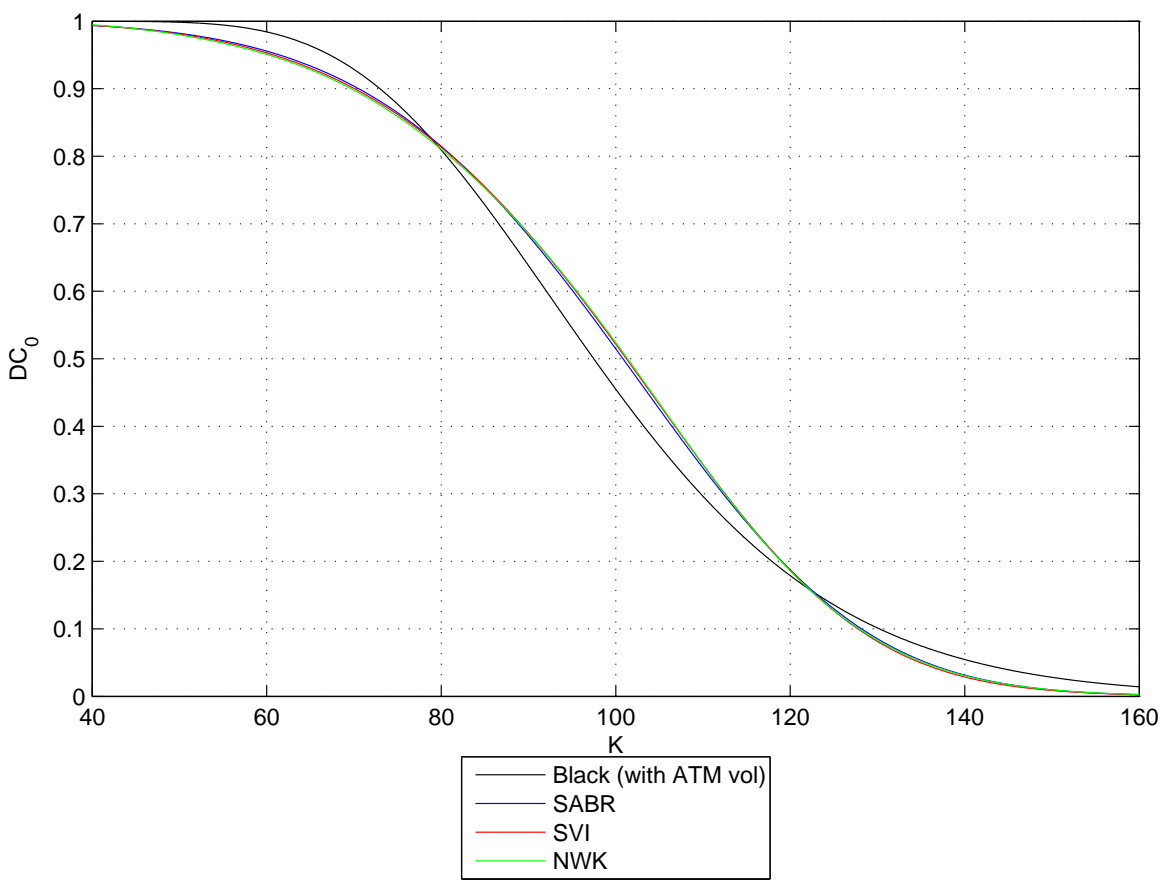

Figure 6: Undiscounted prices of digital calls $(T=1$ year, $F=100)$

For this application, it is interesting to investigate the sensitivity of our method and the risk of error associated with the choice of a smile interpolation technique. As implied volatility market data is discrete in strike, there is uncertainty on the interpolation technique to be used, even after the available parametrizations are calibrated.

Due to this uncertainty, the choice of a technique among the available ones, by itself, introduces model risk in our proposed method to price digital options.

The different aspects of model risk in the area of derivatives pricing were introduced by [10] (DERMAN, 1996). To analyze this risk, we work under the theoretical framework built in [9] 
(CONT, 2006). In this approach model risk is measured as the difference between price bounds obtained when varying the pricing model over a predefined set.

Our set of models, $\mathcal{M}$, is composed of our three available parametrizations of the volatility smile, namely SABR, SVI and Nadaraya-Watson Kernel (NWK).

$$
\mathcal{M}=\{S A B R, S V I, N W K\}
$$

Upper and lower bounds for the undiscounted price of strike $K$ digital call are respectively defined as

$$
\pi^{*}(K)=\max _{M \in \mathcal{M}} \mathbb{Q}_{T}^{M}\left(\left\{S_{T} \geq K\right\}\right) \quad \pi_{*}(K)=\min _{M \in \mathcal{M}} \mathbb{Q}_{T}^{M}\left(\left\{S_{T} \geq K\right\}\right)
$$

where the exponent over $\mathbb{Q}_{T}$ means the model $M \in \mathcal{M}$ has been chosen to compute the probability. In this set-up, the model risk measure for strike $K$ digital call is written

$$
\mu(K)=\pi^{*}(K)-\pi_{*}(K)
$$

Defined that way, model risk can be interpreted as the maximum error made on the price by picking the wrong model out of the set.

Table 2 below gathers prices and model risk results for digital calls struck in, at and out of the money. For a given strike, the model risk figure is, by construction, homogeneous with the prices that are undiscounted and expressed in cents of monetary unit.

\begin{tabular}{|c||c|c|c|c|c|}
\hline Strike & 60 & 80 & 100 & 120 & 140 \\
\hline SABR & 95.57 & 81.47 & 51.48 & 18.73 & 3.15 \\
\hline SVI & 95.31 & 81.35 & 52.17 & 18.67 & 2.87 \\
\hline NWK & 95.08 & 81.05 & 52.41 & 18.6 & 3.08 \\
\hline$\pi^{*}-\pi_{*}$ & 0.49 & 0.42 & 0.93 & 0.14 & 0.28 \\
\hline
\end{tabular}

Table 2: Model risk on prices of digital calls (\%)

Table 2 illustrates that the method is stable but sensitive to the parametrization choice as measured by model risk. It advocates for a careful choice, if any, of the interpolation technique.

As we examine further the results in table 2, model risk appears to be larger for the digital call struck at the money. It can also be noted that pricing with the SABR parametrization always leads to a pricing bound (upper or lower depending on the strike). This last remark does not favor the choice of this technique.

\subsection{Modelling of log-return with a parametric distribution}

In this second application we model $X_{T}$, the time $T$ log-return of $S$ defined as

$$
X_{T}=\ln \left(\frac{S_{T}}{S_{0}}\right)
$$

We first use the results obtained in Part 2 to get a non-parametric density for the logreturn. We then introduce the Normal Inverse Gaussian distribution (NIG hereafter) to model the distribution of $X_{T}$.

Considering that $\phi_{T}$, the risk-neutral density of $S_{T}$, is known and given by (2.21), it is possible to express $f_{n p}$ the non-parametric density of $X_{T}$ as 


$$
f_{n p}(x)=S_{0} e^{x} \phi_{T}\left(S_{0} e^{x}\right) \quad x \in \mathbb{R}
$$

See Appendix A.2 for a proof.

This approach to obtain the risk-neutral density of log-return from the risk-neutral density of asset price is also used in [23] (KERMiche, 2009) and [13] (FigLEWSKI, 2010). Figure 7 presents $f_{n p}$ graphs for different smile construction techniques. Density obtained with Black's model is also plotted as a reference, it is a Gaussian density.

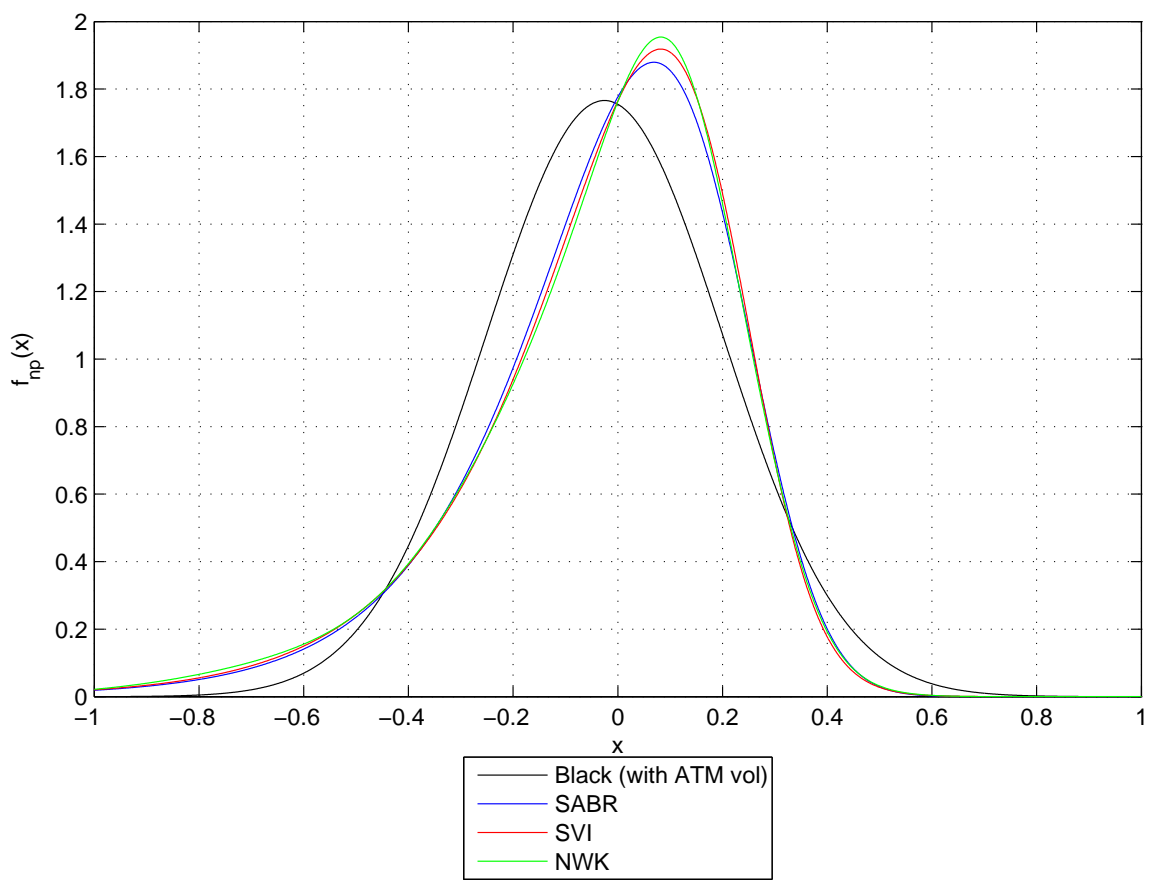

Figure 7: Non-parametric density of log-return

Table 3 presents the values of the first four moments ${ }^{1}$ of $X_{T}$, computed using the method presented in Appendix A.3 and with the non-parametric density $f_{n p}$. Moments of $X_{T}$ in Black's model are also computed to provide a check on the numerical quadrature method.

\begin{tabular}{|c||c|c|c|c|}
\hline & Mean & Variance & Skewness & Kurtosis \\
\hline SABR & -0.0289 & 0.0632 & -1.2086 & 6.7146 \\
\hline SVI & -0.029 & 0.0633 & -1.1421 & 5.5395 \\
\hline NWK & -0.0291 & 0.0632 & -1.0135 & 4.5845 \\
\hline BS & -0.0255 & 0.051 & 0 & 3 \\
\hline
\end{tabular}

Table 3: Moments of $X_{T}$ numerically computed with non-parametric density

To model $X_{T}$ we now use the NIG distribution. It is parametric and we use $f_{n p}$ to determine its parameter set $\theta=(\alpha, \beta, \mu, \delta)$.

The NIG distribution was introduced in [3] (BARNDORFF-NiELSEN, 1997). It allows for the modelling of fat tails, skewness and kurtosis. For applications of the NIG distribution see [21] (Kalemanova, Schmid \& Werner, 2007) where authors use it in the context of CDO $^{2}$ pricing.

\footnotetext{
${ }^{1}$ For a definition of Mean, Variance, Skewness and Kurtosis see Appendix A.3.

${ }^{2}$ A CDO (Collateralized Debt Obligation) is a multi-name credit derivative.
} 
See also [12] (ERIKsson,GHysels \& WANG, 2009) where it is applied to the modelling of the risk-neutral density of an underlying asset price. For details on its properties and implementation see [22] (KALEMANOVA \& WERNER, 2006).

If we suppose the distribution of $X_{T}$ under $\mathbb{Q}_{T}$ to be a NIG, its density $f_{\theta}$ is

$$
f_{\theta}(x)=\frac{\delta \alpha \exp (\delta \gamma+\beta(x-\mu))}{\pi \sqrt{\delta^{2}+(x-\mu)^{2}}} K_{1}\left(\alpha \sqrt{\delta^{2}+(x-\mu)^{2}}\right) \quad x \in \mathbb{R}
$$

$\gamma=\sqrt{\alpha^{2}-\beta^{2}}$ and $K_{1}$ is the second kind modified Bessel function of order 1 , see Chap. 9 in [1] (Abramovitz \& Stegun, 1972).

We want to fit the NIG distribution of $X_{T}$, that is the parameter vector $\theta$, according to the volatility smile considered to be the available market data. It can be done by matching risk-neutral moments, computed numerically using $f_{n p}$. This method takes advantage of the relationship between moments and parameters of the NIG distribution. It is also used, for example, in [12] (ERIKsson, Ghysels \& WANG, 2009).

\begin{tabular}{|c||c|c|c|c|}
\hline & $\alpha$ & $\beta$ & $\mu$ & $\delta$ \\
\hline SABR & 7.1514 & -3.7538 & 0.1431 & 0.2789 \\
\hline SVI & 16.8487 & -12.419 & 0.3301 & 0.3292 \\
\hline NWK & 12.6512 & -8.0654 & 0.2732 & 0.3654 \\
\hline
\end{tabular}

Table 4: NIG parameters (moment matching method)

The results in Table 4 appear to depend on the smile generation technique. This is because the computation of moments is rather sensitive to it. In particular the kurtosis is significantly higher for the SABR parametrization (see Table 3).

Another approach to fit the NIG parameters is to minimize the distance between the distributions, that is the distance between $f_{\theta}$ and $f_{n p}$ seen as elements of the set of probability density functions, with $f_{n p}$ fixed and considered to be the true distribution. This method accounts for the whole distribution but involves an optimization step.

To achieve the fitting we consider three different distance criteria :

the Hellinger distance

$$
D_{H}\left(f_{\theta}, f_{n p}\right)=\sqrt{\frac{1}{2} \int_{-\infty}^{+\infty}\left(\sqrt{f_{\theta}(x)}-\sqrt{f_{n p}(x)}\right)^{2} d x}
$$

the $L_{2}$-norm

$$
L_{2}\left(f_{\theta}, f_{n p}\right)=\sqrt{\int_{-\infty}^{+\infty}\left(f_{\theta}(x)-f_{n p}(x)\right)^{2} d x}
$$

the Relative Entropy (Kullback-Leibler divergence)

$$
D_{K L}\left(f_{n p} \| f_{\theta}\right)=\int_{-\infty}^{+\infty} f_{n p}(x) \ln \left(\frac{f_{n p}(x)}{f_{\theta}(x)}\right) d x
$$

It can be noted that $D_{H}$ and $L_{2}$ are proper distances. $D_{H}$ lies within $[0,1]$ and $L_{2}$ is a member of the $L_{p}-$ norms family.

The Kullback-Leibler divergence $D_{K L}$ has been introduced in information theory. $D_{K L}(F \| G)$ quantifies the additional information needed to describe a reference model $F$, when a model $G$ is given. It is used in probability and statistics as a non-symmetric measure of dissimilarity between distributions. 
The optimization programs to solve are

$\mathcal{P}_{1}: \min _{\theta=(\alpha, \beta, \mu, \delta)} D_{H}\left(f_{\theta}, f_{n p}\right) \quad \mathcal{P}_{2}: \min _{\theta=(\alpha, \beta, \mu, \delta)} L_{2}\left(f_{\theta}, f_{n p}\right) \quad \mathcal{P}_{3}: \min _{\theta=(\alpha, \beta, \mu, \delta)} D_{K L}\left(f_{n p} \| f_{\theta}\right)$

These are non linear minimization problems for a multivariate and real valued objective function, solved with a downhill simplex algorithm. Values shown in Table 4, obtained by moment matching, are used as seed to start the algorithm. Although it makes sense to use this seed, the algorithm is not sensitive to it.

Table 5 shows the distance values associated to the NIG distributions with parameters used as seed of the algorithm. Tables 6,7 and 8 show the NIG parameters obtained after the minimization, for different distance criteria and smile interpolation techniques.

\begin{tabular}{|c||c|c|c|}
\hline & $\mathrm{H}$ & $L_{2}$ & $\mathrm{KL}$ \\
\hline SABR & 0.0424 & 0.0891 & 0.0063 \\
\hline SVI & 0.0042 & 0.0055 & 0.0001 \\
\hline KS & 0.0247 & 0.0495 & 0.0023 \\
\hline
\end{tabular}

Table 5: Distance between non-parametric density and NIG density (parameters in Table 4)

\begin{tabular}{|c||c|c|c|c|}
\hline & $\alpha$ & $\beta$ & $\mu$ & $\delta$ \\
\hline $\mathrm{H}$ & 16.4145 & -11.7119 & 0.3293 & 0.3516 \\
\hline$L_{2}$ & 17.278 & -12.1172 & 0.3438 & 0.3758 \\
\hline KL & 16.0541 & -11.4116 & 0.324 & 0.3493 \\
\hline
\end{tabular}

Table 6: Fitted NIG parameters for SABR parametrization

\begin{tabular}{|c||c|c|c|c|}
\hline & $\alpha$ & $\beta$ & $\mu$ & $\delta$ \\
\hline $\mathrm{H}$ & 15.2555 & -10.9607 & 0.3074 & 0.3256 \\
\hline$L_{2}$ & 15.8999 & -11.6095 & 0.3157 & 0.3231 \\
\hline KL & 15.2107 & -10.92 & 0.3068 & 0.3256 \\
\hline
\end{tabular}

Table 7: Fitted NIG parameters for SVI parametrization

\begin{tabular}{|c||c|c|c|c|}
\hline & $\alpha$ & $\beta$ & $\mu$ & $\delta$ \\
\hline $\mathrm{H}$ & 15.5525 & -11.0935 & 0.316 & 0.3385 \\
\hline$L_{2}$ & 11.4192 & -7.834 & 0.2524 & 0.3023 \\
\hline KL & 14.9733 & -10.6426 & 0.3075 & 0.3332 \\
\hline
\end{tabular}

Table 8: Fitted NIG parameters for NWK technique

Solving the distance minimization problem is fast. To obtain Tables 6,7 and 8 takes 20 seconds. All computations are done with a Matlab program on a personal computer.

Figure 8 shows the risk-neutral NIG densities of log-return obtained after minimizing the Kullback-Leibler divergence for the different smile generation techniques. Local differences observed near their maxima illustrate the sensitivity of the results to the choice of the smile technique. Curves are globally similar to each other which confirm the stability of our approach. 


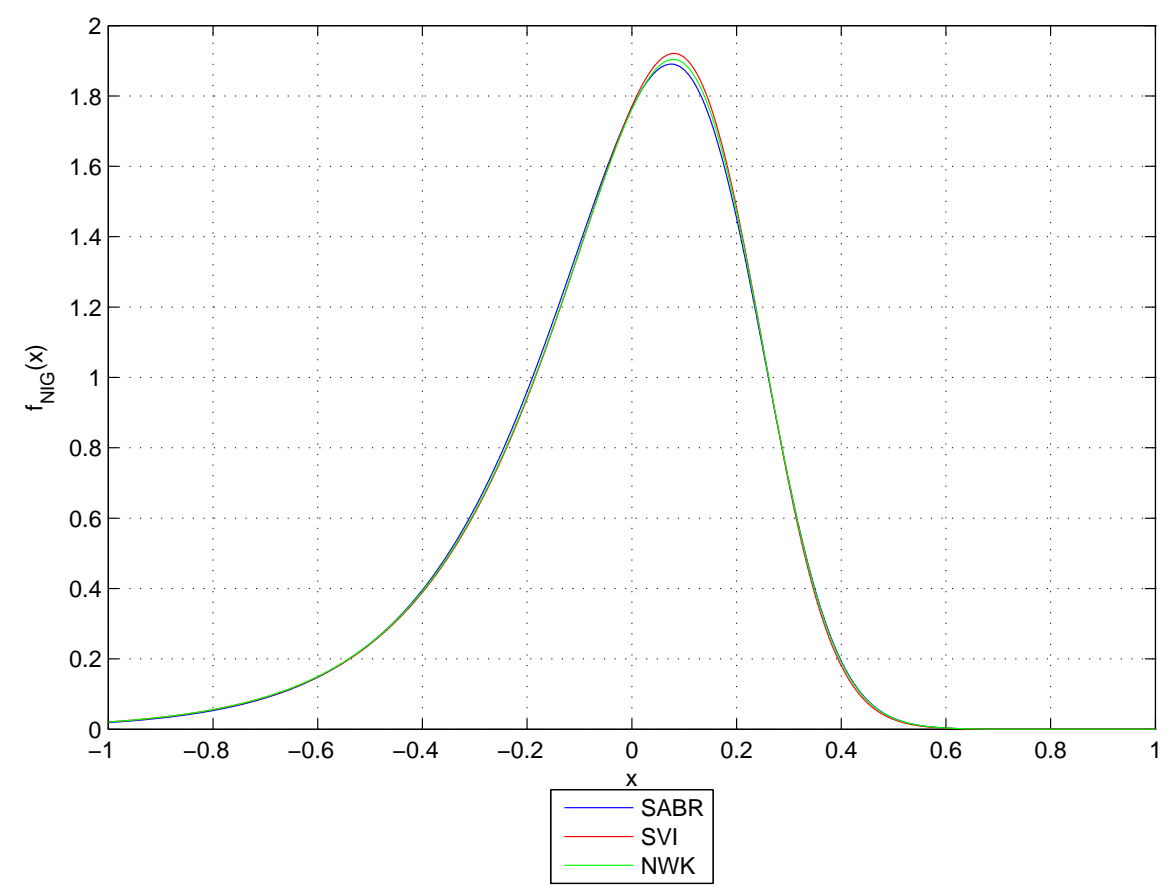

Figure 8: Fitted NIG density of log-return (relative entropy criterion)

\section{Conclusion}

We have proposed in this paper an exact expression of an underlying asset price risk-neutral density as a function of the volatility smile. The proposed expression is a decomposition of the density as a lognormal density, corresponding to Black's model (fitted ATM), plus two adjustment terms accounting for smile level and shape. In the way we have also obtained two no-arbitrage conditions concerning the volatility smile.

The implementation steps of our approach have been detailed and numerical results have been provided in the context of two practical applications. The first one was the pricing of digital options for which model risk has been investigated and the second was the fitting of a NIG distribution for log-return modelling. The proposed method proves to be simple to implement and to perform well on real market data. It turns out to be stable but sensitive to the choice of a smile interpolation technique.

Our methodology to build risk-neutral density appears to be suitable for industrial as well as research purposes. For example, a risk management department can use it to control the valuation of a trading portfolio of European derivatives. It can also be used to obtain marginal distributions when modelling the joint distribution of several underlying assets.

\section{References}

[1] Abramovitz, M. and I.A. Stegun (1972). Handbook of mathematical functions, Dover (10th printing).

[2] Aït-Sahalia, Y. and A. Lo (1998). Nonparametric Estimation of State-Price Densities Implicit in Financial Asset Prices. The Journal of Finance, Vol. 53, No. 2 (Apr., 1998), pp. 499-547

[3] Barndorff-Nielsen, O.E. (1997). Normal Inverse Gaussian distributions and stochastic volatility modelling. Scandinavian Journal of statistics, Vol. 24 (1997), pp. 113 
[4] Black, F. (1976). The Pricing of Commodity Contracts. Journal of Financial Economics, Vol. 3, No. 1, pp. 167-179

[5] Black, F. and M. Scholes (1973). The Pricing of Options and Corporate Liabilities. The Journal of Political Economy, Vol. 81, No. 3 (May - Jun., 1973), pp. 637-654

[6] Bliss, R. and N. Panigirtzoglou (2002). Testing the stability of implied probability density functions. Journal of Banking and Finance, Vol. 26, pp. 381-422

[7] Breeden, D. and R. Litzenberger (1978). Prices of State-Contingent Claims Implicit in Option Prices. The Journal of Business, Vol. 51, No. 4 (Oct., 1978), pp. 621-651

[8] Brunner, B. and R. Hafner (2003). Arbitrage-free estimation of the risk-neutral density from implied volatility smile. The Journal of Computational Finance, 7(1) pp. 75-106

[9] Cont, R. (2006). Model uncertainty and its impact on the pricing of derivative instruments. Mathematical Finance, Vol. 16, No. 3 (July 2006), pp. 519-547

[10] Derman, E. (1996). Model Risk. Goldman Sachs Quantitative Strategies Research Notes (April 1996)

[11] Dupire, B. (1993). Pricing and hedging with smiles. Proceedings of AFFI conference, La Baule, June 1993

[12] Eriksson, A., Ghysels, E. and F. Wang (2009). The Normal Inverse Gaussian Distribution and the Pricing of Derivatives. The Journal of Derivatives, Vol. 16 (Spring 2009), pp. 23-37

[13] Figlewski, S. (2010). Estimating the Implied Risk Neutral Density for the U.S. Market Portfolio. In Volatility and Time Series Econometrics: Essays in Honor of Robert F. Engle, Oxford University Press.

[14] Foata, D. et A. Fuchs (2003). Calcul des Probabilités, Dunod (2 ${ }^{\text {ème }}$ Ed.)

[15] Gatheral, J. (2004). A parsimonious arbitrage free implied volatility parametrization. Global Derivatives conference, Madrid 2004.

[16] Hagan, P., D. Kumar, A. Lesniewski and D. Woodward (2002). Managing smile risk. Wilmott Magazine July 2002, 84-108

[17] Jackwerth, J. (2000). Recovering Risk Aversion from Option Prices and Realized Returns. The Review of Financial Studies, Vol. 13, No. 2 (Summer, 2000), pp. 433-451

[18] Jackwerth, J. (2004). Option-Implied Risk-Neutral Distributions and Risk Aversion. The Research Foundation of the AIMR.

[19] Jackwerth, J. and M. Rubinstein (1996). Recovering Probability Distributions from Option Prices. The Journal of Finance, Vol. 51, No. 5 (Dec., 1996), pp. 1611-1631

[20] Jondeau, E. and M. Rockinger (2000). Reading the smile: the message conveyed by methods which infer risk neutral densities. Journal of International Money and Finance,Vol. 19 (2000), pp. 885-915

[21] Kalemanova, A., Schmid, B. and R. Werner (2007). The Normal Inverse Gaussian Distribution for Synthetic CDO Pricing. Journal of Derivatives, Spring 2007, Vol. 14, No. 3, pp. 80-94

[22] Kalemanova, A. and R. Werner (2006). A short note on the efficient implementation of the Normal Inverse Gaussian distribution. Working Paper

[23] Kermiche, L. (2009). Dynamics of Implied Distributions: Evidence from the CAC 40 Options Market. Finance, 2009, Vol. 30 Issue 2, pp. 63-103 
[24] Lee, R. (2005). Implied Volatility: Statics, Dynamics and Probabilistic Interpretation. In Recent Advances in Applied Probability, Springer.

[25] Merton, R. (1973). Theory of Rational Option Pricing. The Bell Journal of Economics and Management Science, Vol. 4, No. 1 (Spring, 1973), pp. 141-183

[26] Obloj, J. (2008). Fine-tune your smile, correction to Hagan et al., Wilmott Magazine, May/June 2008, pp. 102-109

[27] Portait, R. and P. Poncet (2009). Finance de marché, Dalloz (2 ème Ed.)

[28] Taleb, N. (1997). Dynamic hedging, Managing vanilla and exotic options. John Wiley \& Sons Inc, Wiley Finance Editions.

\section{A Appendix}

\section{A.1 Proof of 2.17 and 2.18}

First recall three usefull identities for greeks calculation

$$
\frac{\partial d_{1}}{\partial K}=\frac{\partial d_{0}}{\partial K} \quad \frac{\partial d_{1}}{\partial \sigma}=\frac{\partial d_{0}}{\partial \sigma}+\sqrt{T} \quad F n\left(d_{1}\right)=K n\left(d_{0}\right)
$$

We then obtain

$$
\begin{gathered}
\frac{\partial C_{B}}{\partial K}=F \frac{\partial \mathcal{N}}{\partial d_{1}} \frac{\partial d_{1}}{\partial K}-K \frac{\partial \mathcal{N}}{\partial d_{0}} \frac{\partial d_{0}}{\partial K}-\mathcal{N}\left(d_{0}\right)=-\mathcal{N}\left(d_{0}\right) \\
\frac{\partial C_{B}}{\partial \sigma}=F \frac{\partial \mathcal{N}}{\partial d_{1}} \frac{\partial d_{1}}{\partial \sigma}-K \frac{\partial \mathcal{N}}{\partial d_{0}} \frac{\partial d_{0}}{\partial \sigma}=\left(F n\left(d_{1}\right)-K n\left(d_{0}\right)\right) \frac{\partial d_{0}}{\partial \sigma}+F n\left(d_{1}\right) \sqrt{T}=n\left(d_{1}\right) F \sqrt{T} \\
\frac{\partial^{2} C_{B}}{\partial K^{2}}=-\frac{\partial \mathcal{N}}{\partial d_{0}} \frac{\partial d_{0}}{\partial K}=\frac{n\left(d_{0}\right)}{K \sigma \sqrt{T}} \\
\frac{\partial^{2} C_{B}}{\partial \sigma \partial K}=-\frac{\partial \mathcal{N}}{\partial d_{0}} \frac{\partial d_{0}}{\partial \sigma}=-n\left(d_{0}\right)\left(-\frac{1}{\sigma^{2}} \frac{\ln \frac{F}{K}}{\sqrt{T}}-\frac{1}{2} \sqrt{T}\right)=\frac{n\left(d_{0}\right) d_{1}}{\sigma} \\
\frac{\partial^{2} C_{B}}{\partial \sigma^{2}}=\frac{\partial n}{\partial d_{1}} \frac{\partial d_{1}}{\partial \sigma} F \sqrt{T}=\left(\frac{1}{\sqrt{2 \pi}}\left(-d_{1}\right) \exp \left(-\frac{d_{1}^{2}}{2}\right)\right)\left(-\frac{1}{\sigma^{2}} \frac{\ln \frac{F}{K}}{\sqrt{T}}+\frac{1}{2} \sqrt{T}\right) F \sqrt{T} \\
=\frac{d_{0} d_{1}}{\sigma} n\left(d_{1}\right) F \sqrt{T}
\end{gathered}
$$

\section{A.2 Expression of the log-return density}

Let $g$ be a monotone and differentiable function and $X$ a random variable with density $f_{X}$. Let $Y$ be the random variable defined as $Y=g(X)$. Its density $f_{Y}$ can be expressed as

$$
f_{Y}: y \longmapsto f_{Y}(y)=\left|\frac{\partial}{\partial y} g^{-1}(y)\right| f_{X}\left(g^{-1}(y)\right)
$$


For details, see Chap. 15 in [14] (FoATA \& FuCHS, 2003).

To obtain (3.4), all you need to do is to apply the above result with $X_{T}$ seen as a transformation of $S_{T}$. That is

$$
X_{T}=g\left(S_{T}\right)
$$

with

$$
\begin{array}{rlrl}
g(x)=\ln \left(\frac{x}{S_{0}}\right) & & x>0 \\
g^{-1}(y) & =S_{0} e^{y} & y & \in \mathbb{R} \\
\frac{\partial}{\partial y} g^{-1}(y) & =S_{0} e^{y} & y & \in \mathbb{R}
\end{array}
$$

so that

$$
f_{n p}(y)=\left|S_{0} e^{y}\right| \phi_{T}\left(S_{0} e^{y}\right)=S_{0} e^{y} \phi_{T}\left(S_{0} e^{y}\right) \quad y \in \mathbb{R}
$$

\section{A.3 Computation of Moments}

Considering a random variable $X$ with $f_{X}$ its density function. Mean, Variance, Skewness and Kurtosis the first four moments are defined, when they exist, as (respectively)

$$
\begin{aligned}
& m=\mathbb{E}[X] \quad v=\mathbb{E}\left[(X-m)^{2}\right] \\
& s=\mathbb{E}\left[\left(\frac{X-m}{\sqrt{v}}\right)^{3}\right] \quad k=\mathbb{E}\left[\left(\frac{X-m}{\sqrt{v}}\right)^{4}\right]
\end{aligned}
$$

If $X$ is Gaussian, $s=0$ and $k=3$. Those values are considered as references when analyzing moments of a distribution.

The computation of $(m, v, s, k)$ relies on the numerical computation of the following integral for $k=1,2,3,4$

$$
I_{k}=\mathbb{E}\left[X^{k}\right]=\int_{0}^{+\infty} x^{k} f_{X}(x) d x
$$

Once the $I_{k}$ are computed for $k=1,2,3,4$, moment values are recovered sequencially using the following identities

$$
\begin{aligned}
m & =I_{1} \\
v & =I_{2}-m^{2} \\
s & =v^{-\frac{3}{2}}\left(I_{3}-3 m v-m^{3}\right) \\
k & =v^{-2}\left(I_{4}-4 m s v^{\frac{3}{2}}-6 m^{2} v-m^{4}\right)
\end{aligned}
$$


Proof.

$$
\begin{aligned}
v & =\mathbb{E}\left[(X-m)^{2}\right]=\mathbb{E}\left[X^{2}-2 X m+m^{2}\right]=I_{2}-m^{2} \\
s & =\mathbb{E}\left[\left(\frac{X-m}{\sqrt{v}}\right)^{3}\right]=v^{-\frac{3}{2}} \mathbb{E}\left[X^{3}-3 X^{2} m+3 X m^{2}-m^{3}\right] \\
& =v^{-\frac{3}{2}}\left(I_{3}-3 m\left(v+m^{2}\right)+2 m^{3}\right)=v^{-\frac{3}{2}}\left(I_{3}-3 m v-m^{3}\right) \\
k & =\mathbb{E}\left[\left(\frac{X-m}{\sqrt{v}}\right)^{4}\right]=v^{-2} \mathbb{E}\left[X^{4}-4 X^{3} m+6 X^{2} m^{2}-4 X m^{3}+m^{4}\right] \\
& =v^{-2}\left(I_{4}-4 m\left(s v^{\frac{3}{2}}+3 m v+m^{3}\right)+6 m^{2}\left(v+m^{2}\right)-3 m^{4}\right) \\
& =v^{-2}\left(I_{4}-4 m s v^{\frac{3}{2}}-6 m^{2} v-m^{4}\right)
\end{aligned}
$$

